\title{
A fully automated system for quantification of knee bone marrow lesions using MRI and the osteoarthritis initiative cohort
}

\author{
Pierre Dodin ${ }^{1}$, François Abram ${ }^{1}$, J ean-Pierre Pelletier², J ohanne Martel-Pelletier ${ }^{2}$ \\ 1. Imaging Research \& Development, ArthroLab Inc., Montreal, Quebec, Canada. 2. Osteoarthritis Research Unit, \\ University of Montreal Hospital Research Centre (CRCHUM), Notre-Dame Hospital, Montreal, Quebec, Canada. \\ Correspondence: Johanne Martel-Pelletier, Osteoarthritis Research Unit, University of Montreal Hospital Research \\ Centre (CRCHUM), Notre-Dame Hospital, 1560 Sherbrooke Street East, Pavillon J. A. de Sève, Y2622, Montreal, Quebec, \\ Canada H2L 4M1. Email: jm@martelpelletier.ca
}

Received: J une 19, 2012

Accepted: September 5, 2012

Online Published: December 17, 2012

DOI : $10.5430 / j b g c . v 3 n 1 p 51$

URL: http://dx.doi.org/10.5430/jbgc.v3n1p51

\section{Abstract}

Background/Objective: Bone marrow lesions (BMLs) have been associated with pain and cartilage degeneration in patients with knee osteoarthritis; their specific detection and quantification is therefore of primary importance. This study aimed at developing a fully automated quantitative BML assessment technology for human knee osteoarthritis using magnetic resonance images (MRI) and two sequences, a T1/T2*-weighted gradient echo (DESS) and a water-sensitive intermediate-weighted turbo spin echo (IW-TSE).

Methods: The automated BML quantification first characterizes the bone and cartilage domains in the DESS sequence using our already published automated technology, then proceeds to the BML quantification which was developed as a four-stage process: selection of structured bright areas corresponding to BMLs, geometric filtering of unrelated structures, segmentation of the BML, and quantification of BML proportion within bone regions. For the IW-TSE sequence, the first step consists of the transfer of the bone and cartilage objects from the DESS to the IW-TSE images, followed by the BML detection and quantification as for the DESS. Validation was performed on 154 OA patients from a subset of the Osteoarthritis Initiative (OAI) cohort (public data sets) in which BML manual segmentation intra- and inter-reader reliability was done for each sequence (DESS and IW-TSE) using the intraclass correlation (ICC). BML comparison between the newly developed automated method with a manual segmentation was performed with ICC for the proportion of BML and Dice similarity coefficient (DSC) for BML localization and geometric extent. Finally, comparisons between the DESS and the IW-TSE sequences were performed for BML incidence and proportion.

Results: Excellent to very good correlations were obtained for both MRI sequences for intra- and inter-reader reliability of the manual BML segmentation. Comparison between the developed automated method and the manual BML segmentation showed excellent to very good correlations in the global knee and regions (ICC $=0.99$ to 0.68 for DESS and 0.99 to 0.77 for IW-TSE sequences) as well as very good to good similarity for the BML geometrical agreement (DESS, 0.60 to 0.41; IW-TSE, 0.59 to 0.41). Data revealed greater BML incidence at the sites of high articular constraints: lateral femoropatellar and medial tibiofemoral articulation. Average BML proportion revealed a scaling factor of about 4.5 -fold more for the IW-TSE compared to the DESS. 
Conclusions: The newly developed fully automated MRI based BML assessment technology not only detects the absence/ presence of these pathological signals in the osteoarthritic human knee, but also provides accurate quantitative assessment of BMLs in the global knee and knee regions. Such automated system will enable large scale studies to be conducted within shorter durations, as well as increase stability of the reading.

\section{Key words}

Automated quantification, Bone marrow lesions, MRI, Knee, Osteoarthritis

\section{I ntroduction}

Osteoarthritis is a common cause of disability in people aged over 60 years. Knee osteoarthritis is a prevalent disease characterized by degenerative changes in all the joint tissues, including hyaline cartilage, synovial membrane, menisci, and subchondral bone. Among the changes in the subchondral bone are lesions referred to as bone marrow lesions (BMLs). In magnetic resonance imaging (MRI), these alterations correspond to a hypersignal in water-sensitive-weighted sequences. The same lesions can also be identified using a number of other MRI sequences, including T1-weighted sequences which reflect a more structural than edematous process. BMLs observed in MRI were found to correlate with pathological bone histologic findings ${ }^{[1]}$ and are important features of human knee osteoarthritis. Hence, studies have established a correlation between BMLs and knee pain ${ }^{[2,3]}$ as well as with cartilage defect progression ${ }^{[4,5]}$ and cartilage volume loss ${ }^{[6,7]}$. Additionally, it has recently been shown that some drugs could reduce the BML concomitantly with improving cartilage volume loss ${ }^{[7,8]}$.

Over time, MRI has proven to be not only a diagnostic tool but also a means for evaluating quantitatively and semiquantitatively various knee joint tissues. In the last decade, scoring of BMLs in MRI systematically appears in the various osteoarthritis knee evaluation systems contributing to the final grade of an overall knee condition ${ }^{[9-13]}$ commonly used in longitudinal studies. In parallel, the combination of MRI and image processing techniques has allowed several semiautomatic and automatic systems to be developed for articular tissue segmentation including bone ${ }^{[14-18]}$, cartilage ${ }^{[19-23]}$, menisci ${ }^{[24]}$, and synovitis ${ }^{[25]}$ for quantitative and semi-quantitative evaluation. However, very few technologies were developed looking at automatically assessing the volume of BMLs. Li et al ${ }^{[26]}$ is the only group, to our knowledge, to report an automated technology for BML segmentation, but their system required both MRI and spectroscopic imaging (MRSI). Driban et al ${ }^{[27]}$ recently described a quantitative method using MRI that relies on manual BML selection. Dijkstra et al ${ }^{[28]}$ developed an MRI semi-automatic solution for BML segmentation using a manual selection of images containing the BML and enhanced with a manual masking of the region of interest.

This study presents a fully automated BML selection and a BML proportion quantification within the knee bone based on MRI. The technology enables the detection of both edema and cysts. The system was developed on two commonly used sequences in knee MRI assessment, a T1/T2-weighted gradient echo (DESS) and a water-sensitive intermediateweighted turbo spin echo (IW-TSE).

\section{MRI acquisitions}

All knee MRI acquisitions were performed as described by the Osteoarthritis Initiative (OAI) cohort protocol using 3T apparatus (Magnetom Trio, Siemens, Erlangen, Germany) at five separate centers ${ }^{[29]}$. The MR protocol included an acquisition using a sagittal DESS sequence (TR/TE, 16.3/4.7 ms; flip angle, 25 degrees; slice thickness/gap, 0.7/0 mm; excitation number, 1 ; matrix size, $384 \times 307$; field of view, $140 \mathrm{~mm}$; resolution, 0.365/0.456 mm; receiver bandwidth, 248 $\mathrm{Hz} /$ pixel; phase direction, A/P). MR images were also acquired using an IW-TSE sequence with sagittal slices (TR/TE, 3200/30 ms; flip angle, 180 degrees; slice thickness/gap, 3/0 mm; excitation number, 1 ; matrix size, $448 \times 313$; field of view, $160 \mathrm{~mm}$; resolution, 0.357/0.511 mm; receiver bandwidth, $248 \mathrm{~Hz} /$ pixel; phase direction, S/I). 


\section{Automatic BML selection and assessment}

The developed fully automated selection and quantification of BML technology consists of four stages following the bone and cartilage automated segmentation in DESS sequence as previously described ${ }^{[17,22]}$ using extra-articular areas for the bone surface. Firstly, an automated selection of structured bright areas corresponding to BMLs inside the subchondral bone was performed. Then, a geometrical filtering in order to discard the noise followed by exclusion of the surrounding bright tissues such as the cartilage and synovial fluid allows the reduction of false detections. The next step consists of the segmentation of the BML. Finally, using the cartilage domain as a reference for the articular domain, the BML is computed as a percentage of the 3D bone object. For the IW-TSE sequence, the first step consists of the transfer of the bone and cartilage objects from the DESS to the IW-TSE images, followed by the BML detection and quantification as for the DESS.

\subsection{DESS sequence}

\subsubsection{Bone computation}

The automated bone segmentation ${ }^{[17]}$ was used in the DESS sequence as a pre-processing for all the MR images. This automated technology operates complete observations of the bone despite bone protuberance or the presence or absence of signals within the bone such as hypersignals (BMLs, enchondromas) and artefacts (magnetic susceptibility, flow). Most situations of signals within the bone from a magnetic susceptibility artefact or presence of a mid-size to large BML are handled by the bone segmentation technology. However, very large BMLs could possibly affect the bone computation. In such a case, the object is detected inside the convex hull of the epiphysis of the bone and an additional process automatically removes the signal from the image and the segmentation of the bone is restarted. Figure 1 shows examples of a very large signal within the bone which was detected and adjusted.

Moreover, in the DESS sequence, the cartilage segmentation previously described ${ }^{[22]}$ provides information on the cartilage object and synovial fluid, which are both needed for the BML selection process.

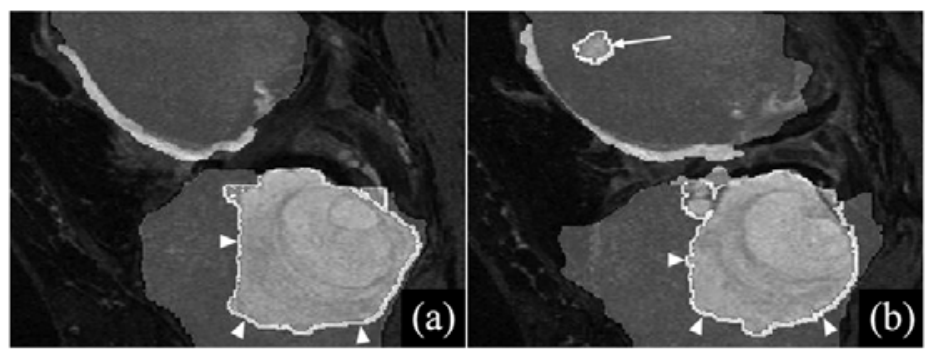

Figure 1. a) and b) Examples of large signals within the tibia preventing proper segmentation of the bone (white arrowheads). In b) the signal in the anterior femoral condyle (white arrow) does not need this additional processing.

\subsubsection{Searching for bright signals inside the bone}

After the bone object is appropriately defined, the automated BML search begins with the detection of bright signals inside the bone.

BMLs consist of two types of lesions: edema, which is characterized by increased signal intensity with a poorly delineated area, and cyst, which also demonstrates increased signal intensity but with a rounded and sharply delineated area ${ }^{[9]}$. In our developed system, these two lesions have been separated into two different classes of signal. The average brightness of the bone as well as that of the cartilage was used to dynamically evaluate the threshold between the edema and cyst with respect to the quality of the image. Cysts have been classified as an image brightness greater than one standard deviation (SD) below the average brightness of the cartilage and are searched with shape constraint, which consists of a minimum 
size filtering and a processing using the mathematical morphology operator "opening” as described ${ }^{[30]}$. The noise signal and the diffuse texture will be discarded by this processing. An edema is classified as an image brightness less intense than the cyst and between the cartilage and the bone brightness and defined as two SD above the average brightness of the bone. There will be no clear boundary or prior known texture and their detection is unconstrained. Figure 2 illustrates the main steps of the processing. From the original image (see Figure 2a), the result of the initial voxel selection based on the computed thresholds (see Figure 2b) showed that inside the bone, the darkest voxels correspond to the range chosen for edema and the lightest to the cyst (see Figure 2c). Based on this processing, there could be false selection due to contrast inhomogeneity. To eliminate false selection due to fat suppression inhomogeneity in the bone, mostly on the femoral condyles at the superior posterior area and on the tibial plateaus at the posterior area, the result is further filtered to remove the areas of less than or equal to $9 \times 9$ voxels. In addition, the selection corresponding to cysts is processed with an opening operation in which the size of the structuring element for the opening operation was set to a square of 2 pixels and is resolution dependent. Of note, these sizes are image resolution-dependent. The final result is illustrated in Figure 2d. Figure 2e shows the final selection of the areas including the bone, cartilage, and the bone edema and cyst. In Figure 3 are other examples of the bright signal selection and differentiation of cysts from surrounding edema detected in different regions of the femoral condyles.

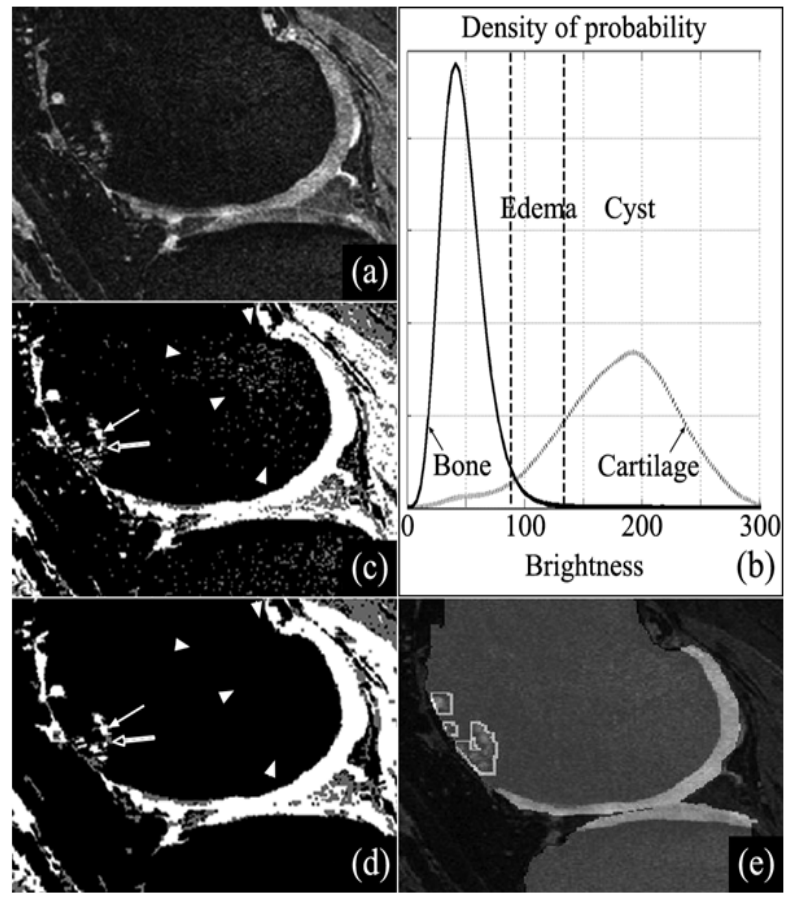

Figure 2. a) Initial DESS image in which prior knowledge of bone and cartilage is obtained by automated segmentation. b) Histogram of the image brightness allowing threshold computation used for voxel selection. The bone probability density (solid black line) and the cartilage probability density (dotted gray line) allow computation of the lower BML threshold (left dashed line) and the threshold (right dashed line) separating edema (lower intensity) from cysts (higher intensity). c) Initial selection of the voxels using the dynamic thresholds: inside the bone, black voxels are non-selected areas, light gray voxels are edema (arrow heads) and white voxels are cysts (arrows). d) Post-processing result: inconsistent false detections (arrowheads in c) are discarded by the filter; only final selected voxels remain (arrows) where black voxels are non-selected areas, light gray voxels are edema (hollow arrow) and white voxels are cysts (solid arrow). e) Final selection of edema and cyst signals within the bone in the context of a full segmentation including bone (dark gray) and cartilage (light gray) objects used for threshold computation. 


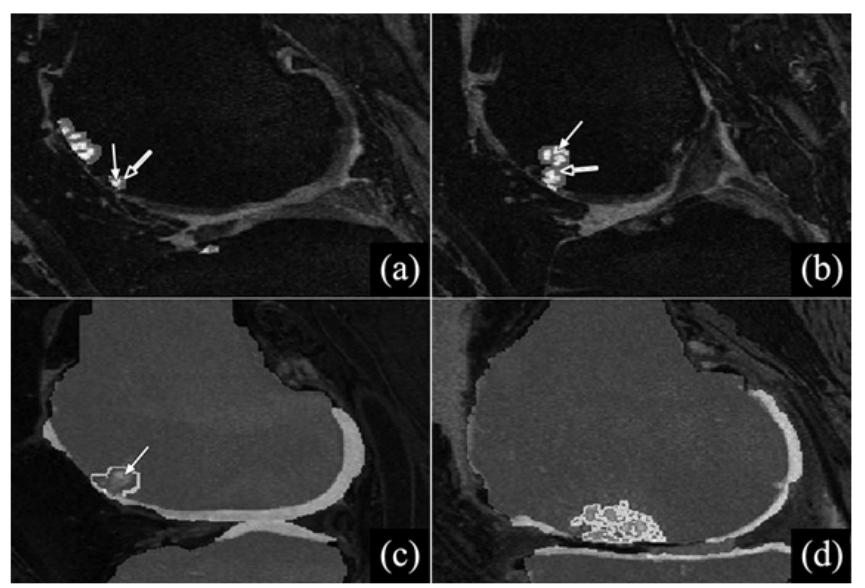

Figure 3. a, b) Cysts (solid arrows) surrounded by edema (hollow arrows) detected in the anterior region of the femoral condyle. c, d) Delineated edema in the anterior lateral and central medial femoral condyle with cysts (solid arrow), bone (dark gray), and cartilage (light gray) objects .

\subsubsection{False detection filtering for final BML selection}

The pre-processing of the bone, smoothing and extension, can cause the bone object to overlap other tissues locally, including signals from neighbouring bright tissues, i.e. cartilage, synovial fluid, and bone protuberance on the vertical walls of the femoral condyles. On the one hand, for the cartilage and synovial fluid, discarding these false selections is done by filtering with prior knowledge of the surrounding bright tissues, which is done using objects provided by the cartilage segmentation from the algorithm previously described ${ }^{[22]}$. On the other hand, as the bone protuberance is close to the boundary of the re-parameterized surface in polar coordinates used in smoothing processes, partial volumes are most likely to generate false detections at this anatomical location. This is prevented by the mathematical morphology operator "erosion" ${ }^{[30]}$ using a structuring element of size equal to twice the image spacing. Hence, if an actual BML extends to the vertical wall, the technology will detect most of its volume except the part excluded by this step at this location. This processing prevents considering a partial volume as a BML.

\subsubsection{BML proportion computation}

For easier transversal inter-patient comparison, data is calculated as the percentage of BML size in the bone object. To this end, in addition to the global knee, the bone was also partitioned into regions ${ }^{[9]}$. The femur epiphysis was divided into 6 regions: anterior, central, and posterior of the medial and lateral femoral condyles (see Figure 4). The tibial plateau was divided into 2 regions: medial and lateral. This is performed using the cartilage domain, permitting a 3D identification of the regions. For the femur cartilage domain regions, the central femoral condyle is defined by the maximum limit, anterior and posterior, of the perpendicular projection of the tibial plateau cartilage domains on the femur object. Additionally, a position $\mathrm{O}$ was defined as the centroid of the 3D cartilage domain (see Figure 4a). Using the point $\mathrm{O}$, a virtual line is automatically computed perpendicularly to the images, and then the 3D boundaries of each region defined in the cartilage domain (see Figure 4b) are projected onto this line for each slice to define a 3D representation of each region. Non-selected parts of the bone are discarded. By limiting the bone object to the epiphysis site for BML evaluation, the volumes of all the bone regions are comparable, obtaining a more uniform normalization of the BML in each region. The sagittal slice located between the lateral and the medial plateau cartilage domain is used to divide the tibial epiphysis into a lateral and a medial 3D object. To expand the analysis to more global areas, regions have been combined as follows. For the femoral condyles, the combination of the anterior/central/posterior in each laterality provides the medial and lateral, and the combination of medial and lateral, the global femoral condyle. For the tibial plateau, combination of the medial and lateral provides the global tibial plateau. The global knee is obtained by the combination of the global femoral condyle and tibial plateau. 


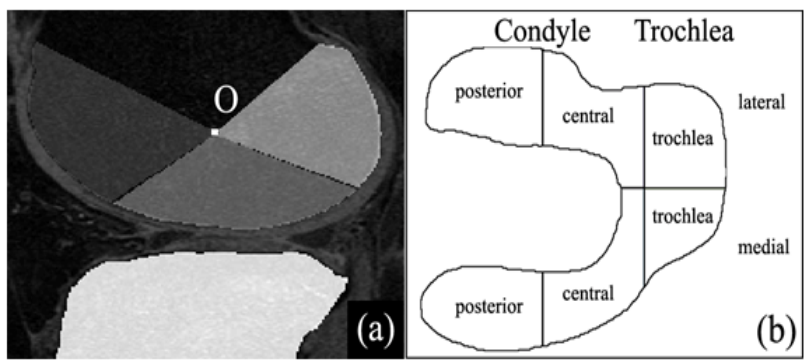

Figure 4. 3D areas shown in the DESS image in a) lateral femoral condyle and b) as computed from femoral cartilage domain sub-regions. Point $\mathrm{O}$ in a) is the reference position.

To determine the BML proportion inside a specific bone region, we used the intersection between the BML and the bone region which provides the number of voxels of the BML which is then divided by the number of voxels of the bone region.

\subsection{W-TSE sequence}

\subsubsection{J oint structure selection}

To perform the BML detection in the IW-TSE images, and since segmentation of bone and cartilage in this sequence is problematic as the bone to cartilage interface is not contrasted enough, the localization of the bone, cartilage, and synovial fluid information obtained in the DESS image are transferred, through the 3D space, to the IW-TSE sequence. This operation assumes that the joint did not move between the acquisitions, avoiding mismatch of the structures in 3D.

\subsubsection{BML selection}

In the IW-TSE, unlike in the DESS image, edema and cyst are more difficult to discriminate by the brightness choice as described above for the DESS. The level of fat suppression is very different between the two MRI sequences; the average bone in IW-TSE is much darker and edema may correspond to a much lesser brightness than in DESS. Thus, in the IW-TSE, the brightness range does not allow a precise discrimination between the two types of signal (cyst and edema). Therefore, the strategy consists of a first detection of the cyst by using the same brightness threshold computation as described above (see Figure 2). Further, the edema pixels are selected as voxels with intensity greater than the average of the bone voxels (see Figure 5). To limit false detections, a filter has been added. Preliminary experiments, using various filtering window sizes, revealed that a $14 \times 14$ voxel window was optimum. This procedure allows the selection of the edema pixels around the cyst area, and permits these two features to be discriminated.

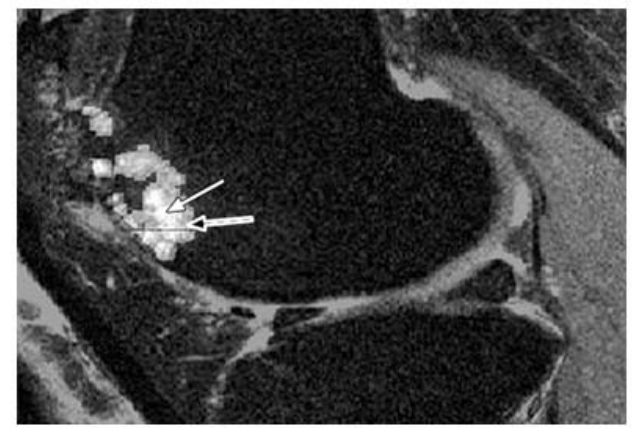

Figure 5. BML voxel selection in the IW-TSE sequence of the cyst (solid arrow) and edema (hollow arrow) areas inside the bone. 


\subsubsection{BML quantification}

The same technique as for the DESS sequence is used to compute the bone partition and the proportion of the BMLs in the bone object.

\section{Validation analysis}

\subsection{Cohort description}

Data used for this study were obtained from the OAI database, which is available at http://www.oai.ucsf.edu/. MR images analyzed are from the OAI sub-cohort 0.1.B (V00IMAGESB SAS Variable) and consist of the first 160 individuals having their 12 month visit and referred to as "progression sub-cohort" as they showed symptomatic and radiologic knee OA at the beginning of the study. For patient inclusion and eligibility criteria, please refer to the section 4.2.2 of the document StudyDesignProtocol and appendix A of the document StudyDesignAppendices. In brief, subjects must show frequent knee symptoms defined as pain, aching or stiffness in or around the knee on most days for at least one month during the past 12 months and radiographic tibiofemoral knee osteoarthritis, defined as definite tibiofemoral osteophytes equivalent to Kellgren and Lawrence grade $\geqslant 2$ on the fixed flexion radiograph.

From this set, 160 left knees were selected as they had both IW-TSE and DESS MRI acquisitions. For our study, additional exclusion criteria included the spatial mismatch of the anatomical structure in the DESS and IW-TSE MRI sequences and the low image quality due to inoperative fat suppression. On this basis, six exams were discarded after quality control. From the 154 images studied, two patients had reconstruction of the anterior cruciate ligament of the left knee and images showed magnetic susceptibility artefacts in the femoral condyles and tibial plateaus. These were kept in the study, as our developed technology handles such situations. One patient had a bone-to-bone tendon graft with a femoral metallic interference screw causing only minor susceptibility artefact (with no distortion of the femorotibial cartilage surfaces) and a tibial resorbable screw causing no artefact at all. The other patient had a hamstring tendon graft tacked at both ends with metallic interference screws causing limited magnetic susceptibility artefacts in the adjacent bony regions with no distortion of the femorotibial cartilage surfaces. Moreover, two other patients showed enchondromas as described ${ }^{[31]}$, one in the femoral condyle and one in the lateral tibial plateau; these patient images were also kept in the study as their lesions were external to the epiphysis and therefore did not interfere with the developed automated process.

\subsection{Validation methodology}

\subsubsection{Statistical analysis}

The validation analysis consists of comparing the newly developed automated BML quantification with manual segmentation quantification ( $\mathrm{n}=154)$. For the manual segmentation, the images were assessed by two expert readers who were trained by a musculoskeletal radiologist and have over 10 years of experience in the field. Intra- and inter-reader assessment was performed on a subset of $55 \mathrm{OAI}$ individual images for each MRI sequence.

For the comparison, the BML edema and cyst were computed together. Firstly, the incidence of patients showing BML in a region was evaluated. Since the BML marker is computed on a continuous scale, the intraclass correlation (ICC) was used for the intra- and inter-reader analyses as well as for the comparison of the BML proportion between the manual and the developed automated technology. Finally, the Dice similarity coefficient (DSC) allowing geometric comparison of the segmented BML objects between the manual and automated segmentation was performed. This latter expresses the geometrical correspondence of the selected objects from DSC $=1$ identical object, to DSC $=0$ completely disjoined objects. In the literature, it is stated that a DSC value of 0.7 represents an excellent agreement ${ }^{[28,32]}$. 


\subsubsection{Manual segmentation}

For the manual BML proportion evaluation, the DESS or IW-TSE sequences are opened using the DICOM compliant software DICOMWORKS ${ }^{[33]}$ where the image contrast is set for BML reading, then exported in TIFF format and opened in image processing software (Adobe Photoshop, San-Jose, CA). Each edema and cyst is delineated separately in a specific colour channel using selection tools. After saving, the TIFF images and corresponding DICOM sequences are opened in computational software (Matlab, Mathworks, Natick, MA) enabling the conversion of pixels into a 3D object, using acquisition resolution. The BML object is then expressed as percentage of bone volume, as described above for the automated system. The analyses were done for the global knee and each region as described above.

\section{Results}

\subsection{I ntra- and inter-reader reliability}

Intra-reader reliability of the manual BML segmentation for both DESS and IW-TSE ranges from excellent to very good (see Table 1). For the DESS sequence values were 0.89 for the global knee, 0.91 for the femoral condyle, and 0.97 for the tibial plateau. For the IW-TSE sequence, values of $0.83,0.80$, and 0.76 were obtained, respectively. The inter-reader reliability was also excellent to very good, ranging for DESS from 0.99 to 0.73 and for IW-TSE from 0.93 to 0.77 (see Table 2).

Table 1. Intra-reader ICC correlation

\begin{tabular}{|c|c|c|}
\hline BONE REGION & DESS & IW-TSE \\
\hline \multicolumn{3}{|l|}{ FEMORAL CONDYLE } \\
\hline Medial & 0.90 & 0.80 \\
\hline Medial Anterior & 0.95 & 0.85 \\
\hline Medial Central & 0.91 & 0.83 \\
\hline Medial Posterior & 0.92 & 0.97 \\
\hline Lateral & 0.91 & 0.90 \\
\hline Lateral Anterior & 0.93 & 0.91 \\
\hline Lateral Central & 0.90 & 0.75 \\
\hline Lateral Posterior & 0.90 & 0.97 \\
\hline Medial+L ateral Anterior & 0.93 & 0.90 \\
\hline Medialt & 0.90 & 0.80 \\
\hline Medial+Lateral Posterior & 0.88 & 0.97 \\
\hline \multicolumn{3}{|l|}{ TIBIAL PLATEAU } \\
\hline Medial & 0.92 & 0.85 \\
\hline Lateral & 0.98 & 0.82 \\
\hline \multicolumn{3}{|l|}{ GLOBAL } \\
\hline Femoral Condyle & 0.91 & 0.80 \\
\hline Tibial Plateau & 0.97 & 0.76 \\
\hline Knee & 0.89 & 0.83 \\
\hline
\end{tabular}


Table 2. Inter-reader ICC correlation

\begin{tabular}{lll}
\hline BONE REGION & DESS & IW-TSE \\
\hline FEMORAL CONDYLE & & 0.81 \\
Medial & 0.92 & 0.84 \\
Medial Anterior & 0.92 & 0.80 \\
Medial Central & 0.94 & 0.77 \\
Medial Posterior & 0.84 & 0.87 \\
Lateral & 0.93 & 0.87 \\
Lateral Anterior & 0.93 & 0.77 \\
Lateral Central & 0.73 & 0.93 \\
Lateral Posterior & 0.90 & 0.87 \\
Medial+Lateral Anterior & 0.93 & 0.78 \\
Medial+Lateral Central & 0.91 & 0.79 \\
Medial+Lateral Posterior & 0.86 & \\
TIBIAL PLATEAU & & 0.90 \\
Medial & 0.99 & 0.90 \\
Lateral & 0.99 & 0.83 \\
GLOBAL & & 0.92 \\
Femoral Condyle & 0.93 & \\
Tibial Plateau & 0.99 & 0.99 \\
Knee & &
\end{tabular}

\subsection{Comparison between the manual and automated BML proportion}

For both sequences, the ICC between manual and automated BML proportion was excellent to very good for all regions (DESS, 0.99-0.68; IW-TSE, 0.99-0.77) (see Table 3). This demonstrates the correspondence between the manual results and the automated results in terms of presence of BMLs as well as in terms of values of the BML proportion.

Table 3. Manual and automated intraclass correlation

\begin{tabular}{lll}
\hline BONE REGION & DESS & IW-TSE \\
\hline FEMORAL CONDYLE & & \\
Medial & 0.96 & 0.82 \\
Medial Anterior & 0.85 & 0.93 \\
Medial Central & 0.95 & 0.79 \\
Medial Posterior & 0.74 & 0.85 \\
Lateral & 0.95 & 0.88 \\
Lateral Anterior & 0.96 & 0.85 \\
Lateral Central & 0.80 & 0.95 \\
Lateral Posterior & 0.68 & 0.99 \\
Medial+Lateral Anterior & 0.95 & 0.85 \\
Medial+Lateral Central & 0.95 & 0.78 \\
Medial+Lateral Posterior & 0.71 & 0.87 \\
TIBIAL PLATEAU & & \\
Medial & 0.99 & 0.83 \\
Lateral & 0.99 & 0.79 \\
GLOBAL & &
\end{tabular}




\subsection{Dice similarity coefficient between manual and automated BML objects}

The DSC computed between the BML objects obtained by manual and automated BML selection reveals very good to good coefficients for both sequences (see Table 4), indicating an agreement between BML 3D localization and geometrical extent.

Table 4. DSC between manual and automated BML selections

\begin{tabular}{|c|c|c|}
\hline BONE REGION & DESS & IW-TSE \\
\hline \multicolumn{3}{|l|}{ FEMORAL CONDYLE } \\
\hline Medial & 0.48 & 0.47 \\
\hline Medial Anterior & 0.60 & 0.59 \\
\hline Medial Central & 0.50 & 0.45 \\
\hline Medial Posterior & 0.43 & 0.43 \\
\hline Lateral & 0.52 & 0.49 \\
\hline Lateral Anterior & 0.56 & 0.48 \\
\hline Lateral Central & 0.41 & 0.49 \\
\hline Lateral Posterior & 0.44 & 0.47 \\
\hline Medial+Lateral Anterior & 0.57 & 0.49 \\
\hline Medial+Lateral Central & 0.47 & 0.46 \\
\hline Medial+Lateral Posterior & 0.44 & 0.43 \\
\hline \multicolumn{3}{|l|}{ TIBIAL PLATEAU } \\
\hline Medial & 0.40 & 0.57 \\
\hline Lateral & 0.59 & 0.41 \\
\hline \multicolumn{3}{|l|}{ GLOBAL } \\
\hline Femoral Condyle & 0.51 & 0.49 \\
\hline Tibial Plateau & 0.42 & 0.54 \\
\hline Knee & 0.49 & 0.52 \\
\hline
\end{tabular}

\subsection{I ncidence of BML: manual segmentation}

Since manually segmented BML is considered the gold standard for volumetric assessment, we calculated the respective incidence of patients showing BMLs in the specified regions in DESS and IW-TSE sequences (see Table 5). Data showed that, with regard to the regions in the femoral condyles and tibial plateaus, the highest incidences were in the central (30\%, $31 \%$ of the 154 patients for DESS and IW-TSE respectively) and medial femoral condyle (27\%, 25\%) and in the medial tibial plateau (16\%, 24\%), while the lowest incidence was in the lateral posterior femoral condyle (4\% for both) and the lateral tibial plateau (5\%,6\%). These data show that the greater BML incidences appear at the sites where articular constraints are high, the lateral femoropatellar articulation and the medial tibiofemoral articulation. For combined regions, data showed that the highest presence of BMLs was in the global knee with 56\% and 48\% for the DESS and IW-TSE respectively, followed by the global femoral condyle in which $49 \%$ and $40 \%$ were found. These suggest that, for a longitudinal or cross-sectional study, it would be preferable to use larger regions to preserve the statistical power. 
Table 5. Incidence of patients amongst the 154 patients showing BMLs in each region

\begin{tabular}{lll}
\hline BONE REGION & DESS & IW-TSE \\
\hline FEMORAL CONDYLE & & 38 \\
Medial & 41 & 9 \\
Medial Anterior & 10 & 31 \\
Medial Central & 31 & 16 \\
Medial Posterior & 16 & 35 \\
Lateral & 46 & 32 \\
Lateral Anterior & 35 & 22 \\
Lateral Central & 22 & 6 \\
Lateral Posterior & 6 & 35 \\
Medial+Lateral Anterior & 41 & 47 \\
Medial+Lateral Central & 46 & 22 \\
Medial+Lateral Posterior & 22 & \\
TIBIAL PLATEAU & & 37 \\
Medial & 25 & 10 \\
Lateral & 7 & \\
GLOBAL & & 61 \\
Femoral Condyle & 76 & 44 \\
Tibial Plateau & 31 & 74 \\
Knee & 87 & \\
\hline
\end{tabular}

\subsection{Comparison of manual BML proportion between DESS and I W-TSE sequences}

Table 6. Average manual BML proportion (\%) in DESS and IW-TSE

\begin{tabular}{lll}
\hline BONE REGION & DESS & IW-TSE \\
\hline FEMORAL CONDYLE & & \\
Medial & 0.9 & 4.0 \\
Medial Anterior & 0.8 & 7.5 \\
Medial Central & 2.4 & 9.2 \\
Medial Posterior & 0.6 & 5.6 \\
Lateral & 1.0 & 6.0 \\
Lateral Anterior & 3.3 & 14.1 \\
Lateral Central & 0.6 & 4.2 \\
Lateral Posterior & 0.7 & 3.6 \\
Medial+Lateral Anterior & 1.7 & 8.6 \\
Medial+Lateral Central & 1.0 & 4.0 \\
Medial+Lateral Posterior & 0.3 & 2.5 \\
TIBIAL PLATEAU & & \\
Medial & 1.9 & 4.6 \\
Lateral & 1.8 & 2.3 \\
GLOBAL & & \\
Femoral Condyle & 0.5 & 3.0 \\
Tibial Plateau & 0.9 & 2.0 \\
Knee & 0.4 & 1.8 \\
\hline
\end{tabular}


The BML proportion obtained by DESS and IW-TSE sequences (see Table 6) revealed that the greater sizes in DESS as well as in IW-TSE are in the femoral condyles at the lateral anterior area followed by the medial central area which corroborates with the incidence analysis. Of note, a scaling factor of about 4.5-fold more was found for the IW-TSE compared to the DESS.

\section{Discussion}

BMLs are frequently associated with osteoarthritis and are considered important markers of the disease development and progression. Despite the fact that these changes play a major role in this disease, semi-quantitative scoring systems ${ }^{[9-13]}$ are generally used because a reliable quantification method is lacking. There have been very few technologies developed aimed at the quantification of BMLs using solely MRI. Here we report the development and validation of a novel and fully automated method for the segmentation and quantification of BMLs in knee osteoarthritis patients, discriminating cyst from edema. This new system allows the quantification of BMLs not only in the global knee, but also in the femoral condyles and tibial plateaus independently as well as for different knee regions. Validation experiments comparing the developed automated method with the manual BML segmentation, revealed excellent to very good values in terms of accuracy.

Compared to the other published works ${ }^{[26-28]}$, our developed technology provides the advantage of being fully automated. The Dijkstra et al ${ }^{[28]}$ solution is the most similar to the technology reported here as it uses MR images only; however, their method employed manual selection of images containing the BMLs as well as manual masking of the region of interest. The technology described herein automatically processes the bone followed by the identification and quantification of the BMLs. The main difficulty in automatically selecting the BML was to handle the artefacts at the proximity of the bone. These included partial volumes, truncation artefacts, proximity of synovial fluid, and participant movement, which were solved by relying on a robust bone segmentation technology ${ }^{[17]}$, the use of mathematic morphology operators, and the filtering of unrelated structures using cartilage and synovial fluid information from cartilage segmentation ${ }^{[22]}$. The success of this approach is reflected by the validation study showing an excellent correlation between manual and automated BML selection. In addition, the DSC values which range from 0.60-0.40 are also comparable to those obtained by Dijkstra et al ${ }^{[28]}$ of $0.70-0.53$ using T2-weighted spectral presaturation with inversion recovery (SPIR) and proton density (PD) sequences. However, the putative lower DSC found for our method compared to the method of Dijkstra et al ${ }^{[28]}$ can be explained by the fact that their solution involves a manual spatial identification of the BMLs before segmentation, thus increasing the optimal correspondence with the manual segmentation. This also indicates that using the manual segmentation as a reference for the validation study with fully automated technology could be a limitation as, although manual segmentation is currently considered the gold standard for the diagnosis of BMLs (e.g. absence, mild, medium, severe), several concerns prevent it from being the definite gold standard for BML segmentation. These include the contrast adjustment which may produce an over- or under-segmentation by a slight change in the image contrast, the partial volume which may blur the boundary of the BML making it difficult to localize the BML's real boundary, and the BML's complex shape, which may be difficult to capture by drawing a line with a pointing device.

The choice of adaptive and dynamic thresholding and mathematical morphology filtering allows our technology to overcome problems specifically related to variable signal to noise ratio between the bone regions. This includes difficulties in the reading of femoral condyles in the medial anterior area in the presence of a high volume of synovial fluid in the DESS sequence and in the posterior where the flux artefact is significant in the IW-TSE sequence. The present technical choice also challenges the similarity of the signal between edema and cyst in the IW-TSE sequence allowing discrimination between these two types of lesions. Our data showing a scaling factor of 4.5-fold more for the IW-TSE compared to the DESS concurs with those obtained with another methodology ${ }^{[9]}$ in which BMLs were manually assessed using the WORMS scoring system. 
A potential limitation of the BML assessment in the IW-TSE sequence is the absence of registration between the DESS and IW-TSE sequences to overcome a possible repositioning of the patient between the two acquisitions. MRIs from patients in this situation were excluded from the study. As the nature of the signals between these two sequences is different, a registration will require image distance evaluation based on mutual-information, comparable to the registration performed between CT-scan and PET-scan. Studies are underway to explore solutions to this problem.

The advantage of an automated technology, besides being robust to a range of signal quality, is the improved stability of the reading process as well as being adaptive to the image quality and independent of a specific intensity and/or contrast. Specifically for BML assessment, many issues may impact the results of manual and semi-automated segmentation methods and as mentioned above include contrast, intensity, tuning for the image display, and the drawing of the complex shape of these structures, all of which have an important influence on the final segmentation results and will decrease the stability and the accuracy of the data. Another advantage for clinical trials is that the automated BML quantification could be done in parallel with the automated evaluation of the bone ${ }^{[17]}$ and the cartilage ${ }^{[22]}$, thus saving time. More specifically for the BML reading, the processing time is also an advantage. By using a $3 \mathrm{GHz}$ processor with 2Go of RAM, the BML evaluation is about 12 minutes for both DESS and IW-TSE sequences and is constant whether or not more and larger BMLs are found within a visit. In comparison, a manual BML segmentation is about 20 minutes for the DESS and 10 minutes for the IW-TSE, but could be longer depending on the number and volume of BMLs. Of great interest is that such automated software can be run in parallel on a multicore processor, providing several results in the same amount of time. Finally, the system could work around the clock allowing for the reliable reading of a large number of MRI exams in a short period of time representing a significant advantage particularly in studies inclusive of a large number of patients such as clinical trials.

In conclusion, this new automated technology enables BML detection as well as an innovative quantitative assessment of the BML volume in various regions of the osteoarthritis knee, using routine MRI sequences for knee imaging: DESS T1/T2* weighted and IW-TSE intermediate weighted. A primary advantage of this automated segmentation technology is the possibility of intensive and autonomous computation, enabling images from a large cohort of patients to be analyzed reliably in a shorter time and, more importantly, increased stability of the reading, hence increased accuracy of results. It will allow for a better understanding of the evolution of BMLs and their role during the osteoarthritis process, as well as establishing a correlation with other markers of this disease. It will also help to monitor the effect of treatment on the evolution of BMLs. Lastly, the benefits of such technology could be distinctive whether it is used for clinical studies, clinical diagnosis, or even for surgery. Indeed, in addition to the advantages listed above for clinical studies, the technology provides access to a BML 3D object and localization, which could allow the physician to a more accurate diagnosis and for joint surgery, could assist in the planning of a surgery.

\section{Acknowledgments}

The authors thank André Pelletier and Josée Thériault for their expertise in MRI reading and Dr Marie-Josée Berthiaume, a musculoskeletal radiologist, for the training of AP and JT in reading the BML in the MRI images from both DESS and IW-TSE sequences.

\section{Competing interests}

JPP and JMP are consultants and shareholders of ArthroLab Inc. PD and FA are employees of ArthroLab Inc.

\section{Authors' contributions}

All authors contributed to the conception and design of the study, the acquisition, analysis and interpretation of data, and the drafting and approval of the manuscript. 


\section{References}

[1] Zanetti M, Bruder E, Romero J, et al. Bone marrow edema pattern in osteoarthritic knees: correlation between MR imaging and histologic findings. Radiology. 2000; 215(3):835-40. Available from: http://www.ncbi.nlm.nih.gov/pubmed/10831707

[2] Felson DT, McLaughlin S, Goggins J, et al. Bone marrow edema and its relation to progression of knee osteoarthritis. Ann Intern Med. 2003; 139:330-6. PMid: 12965941

[3] Zhai G, Blizzard L, Srikanth V, et al. Correlates of knee pain in older adults: Tasmanian Older Adult Cohort Study. Arthritis Rheum. 2006; 55(2):264-71. Available from: http://www.ncbi.nlm.nih.gov/pubmed/16583417

[4] Wluka AE, Wang Y, Davies-Tuck M, et al. Bone marrow lesions predict progression of cartilage defects and loss of cartilage volume in healthy middle-aged adults without knee pain over 2 yrs. Rheumatology (Oxford). 2008; 47(9):1392-6. Available from: http://www.ncbi.nlm.nih.gov/pubmed/18606620

[5] Dore D, Martens A, Quinn S, et al. Bone marrow lesions predict site-specific cartilage defect development and volume loss: a prospective study in older adults. Arthritis Res Ther. 2010; 12(6):R222. PMid: 3046535. Available from: http://www.ncbi.nlm.nih.gov/pubmed/21190554

[6] Raynauld JP, Martel-Pelletier J, Berthiaume MJ, et al. Correlation between bone lesion changes and cartilage volume loss in patients with osteoarthritis of the knee as assessed by quantitative magnetic resonance imaging over a 24-month period. Ann Rheum Dis. 2008; 67(5):683-8. Available from: http://www.ncbi.nlm.nih.gov/pubmed/17728333

[7] Wildi LM, Raynauld JP, Martel-Pelletier J, et al. Relationship between bone marrow lesions, cartilage loss and pain in knee osteoarthritis: results from a randomised controlled clinical trial using MRI. Ann Rheum Dis. 2010; 69(12):2118-24. Available from: http://www.ncbi.nlm.nih.gov/pubmed/20610445

[8] Wildi LM, Raynauld JP, Martel-Pelletier J, et al. Chondroitin sulphate reduces both cartilage volume loss and bone marrow lesions in knee osteoarthritis patients starting as early as 6 months after initiation of therapy: a randomised, double-blind, placebo-controlled pilot study using MRI. Ann Rheum Dis. 2011; 70(6):982-9. PMid: 3086081. Available from: http://www.ncbi.nlm.nih.gov/pubmed/21367761

[9] Peterfy CG, Guermazi A, Zaim S, et al. Whole-Organ Magnetic Resonance Imaging Score (WORMS) of the knee in osteoarthritis. Osteoarthritis Cartilage. 2004; 12(3):177-90. Available from: http://www.ncbi.nlm.nih.gov/pubmed/14972335

[10] Kornaat PR, Ceulemans RY, Kroon HM, et al. MRI assessment of knee osteoarthritis: Knee Osteoarthritis Scoring System (KOSS)--inter-observer and intra-observer reproducibility of a compartment-based scoring system. Skeletal Radiol. 2005; 34(2): 95-102. Available from: http://www.ncbi.nlm.nih.gov/pubmed/15480649

[11] Kubassova O, Boyle RD, Pyatnizkiy M. Bone Segmentation in Metacarpophalangeal MR Data. Proceedings of the Third International Conference on Advances in Pattern Recognitoin (ICAPR). 2005; 726-35.

[12] Hunter DJ, Lo GH, Gale D, et al. The reliability of a new scoring system for knee osteoarthritis MRI and the validity of bone marrow lesion assessment: BLOKS (Boston Leeds Osteoarthritis Knee Score). Ann Rheum Dis [Internet]. 2008; 67(2):206-11. Available from: http://www.ncbi.nlm.nih.gov/pubmed/17472995

[13] Hunter DJ, Guermazi A, Lo GH, et al. Evolution of semi-quantitative whole joint assessment of knee OA: MOAKS (MRI Osteoarthritis Knee Score). Osteoarthritis Cartilage [Internet]. 2011; 19(8):990-1002. Available from: http://www.ncbi.nlm.nih.gov/pubmed/21645627

[14] Lorigo LM, Faugeras O, Grimson WEL, et al. Segmentation of bone in clinical knee MRI using texture-based geodesic active contours. Lecture Notes in Computer Science, Proceedings of the First International Conference on Medical Image Computing and Computer-Assisted Intervention. 1998; 1496:1195-204.

[15] Dalvi R, Abugharbieh R, Wilson D, et al. Multi-contrast MR for enhanced bone imaging and segmentation. Conf Proc IEEE Eng Med Biol Soc. 2007; 2007:5620-3. PMid: 19163936 Available from: http://www.ncbi.nlm.nih.gov/pubmed/18003287

[16] Fripp J, Crozier S, Warfield SK, et al. Automatic segmentation of the bone and extraction of the bone-cartilage interface from magnetic resonance images of the knee. Phys Med Biol. 2007; 52(6):1617-31. PMid: 18044568. Available from: http://www.ncbi.nlm.nih.gov/pubmed/17327652

[17] Dodin P, Martel-Pelletier J, Pelletier JP, et al. A fully automated human knee 3D MRI bone segmentation using the ray casting technique. Med Biol Eng Comput. 2011; 49(12):1413-24. Available from: http://www.ncbi.nlm.nih.gov/pubmed/22038239

[18] Tamez-Pena JG, Farber J, Gonzalez PC, et al. Unsupervised segmentation and quantification of anatomical knee features: data from the Osteoarthritis Initiative. IEEE Trans Biomed Eng. 2012; 59(4):1177-86. Available from: http://www.ncbi.nlm.nih.gov/pubmed/22318477

[19] Kauffmann C, Gravel P, Godbout B, et al. Computer-aided method for quantification of cartilage thickness and volume changes using MRI: validation study using a synthetic model. IEEE Trans Biomed Eng. 2003; 50(8):978-88. Available from: http://www.ncbi.nlm.nih.gov/pubmed/12892325 
[20] Folkesson J, Dam E, Olsen OF, et al. Automatic segmentation of the articular cartilage in knee MRI using a hierarchical multi-class classification scheme. Med Image Comput Comput Assist Interv. 2005; 8(Pt. 1): 327-34. Available from: http://www.ncbi.nlm.nih.gov/pubmed/16685862

[21] Stachowiak GP, Stachowiak GW, Podsiadlo P. Automated classification of articular cartilage surfaces based on surface texture. Proc Inst Mech Eng H. 2006; 220(8):831-43. Available from: http://www.ncbi.nlm.nih.gov/pubmed/17236517

[22] Dodin P, Pelletier JP, Martel-Pelletier J, et al. Automatic Human Knee Cartilage Segmentation from 3D Magnetic Resonance Images. IEEE Trans Biomed Eng. 2010; 57: 2699-711. Available from: http://www.ncbi.nlm.nih.gov/pubmed/20639173

[23] Fripp J, Crozier S, Warfield SK, et al. Automatic segmentation and quantitative analysis of the articular cartilages from magnetic resonance images of the knee. IEEE Trans Med Imaging. 2010; 29(1):55-64. Available from: http://www.ncbi.nlm.nih.gov/pubmed/19520633

[24] Swanson MS, Prescott JW, Best TM, et al. Semi-automated segmentation to assess the lateral meniscus in normal and osteoarthritic knees. Osteoarthritis Cartilage. 2010; 18(3):344-53. PMid: 2826568. Available from: http://www.ncbi.nlm.nih.gov/pubmed/19857510

[25] Li W, Abram F, Pelletier JP, et al. Fully automated system for the quantification of human osteoarthritic knee joint effusion volume using magnetic resonance imaging. Arthritis Res Ther. 2010; 12(5): R173. Available from: http://www.ncbi.nlm.nih.gov/pubmed/20846392

[26] Li X, Ma BC, Bolbos RI, et al. Quantitative assessment of bone marrow edema-like lesion and overlying cartilage in knees with osteoarthritis and anterior cruciate ligament tear using MR imaging and spectroscopic imaging at 3 Tesla. J Magn Reson Imaging. 2008; 28(2):453-61. PMid: 2666932. Available from: http://www.ncbi.nlm.nih.gov/pubmed/18666183

[27] Driban JB, Lo GH, Lee JY, et al. Quantitative bone marrow lesion size in osteoarthritic knees correlates with cartilage damage and predicts longitudinal cartilage loss. BMC Musculoskelet Disord. 2011; 12: 217. PMid: 3190349. Available from: http://www.ncbi.nlm.nih.gov/pubmed/21961433

[28] Dijkstra AJ, Anbeek P, Yang KGA, et al. Validation of a novel semiautomated segmentation method for MRI detection of cartilage-related bone marrow lesions. Cartilage. 2010; 1(4): 328-34. http://dx.doi.org/10.1177/1947603510376819

[29] Peterfy CG, Schneider E, Nevitt M. The osteoarthritis initiative: report on the design rationale for the magnetic resonance imaging protocol for the knee. Osteoarthritis Cartilage. 2008; 16(12): 1433-41. PMid: 3048821. Available from: http://www.ncbi.nlm.nih.gov/pubmed/18786841

[30] Soille P. Morphological Image Analysis: Principles and Applications. Springler-Verlag. 1999; 3-540-65671-5.

[31] Walden MJ, Murphey MD, Vidal JA. Incidental enchondromas of the knee. AJR Am J Roentgenol. 2008; 190(6): 1611-5. Available from: http://www.ncbi.nlm.nih.gov/pubmed/18492914

[32] Dice L. Measures of the amount of ecologic association between species. Ecology. 1945; 26: 297-302. http://dx.doi.org/10.2307/1932409

[33] Puech PA, Boussel L, Belfkih S, et al. DicomWorks: software for reviewing DICOM studies and promoting low-cost teleradiology. J Digit Imaging. 2007; 20(2): 122-30. PMid: 3043902. Available from: http://www.ncbi.nlm.nih.gov/pubmed/17333414 